\title{
A comprehensive approach to the photochemical synthesis of bioactive compounds by the reaction of oxazolidine, thiazolidine and pyrazolidine derivatives with indol-2,3-diones
}

\author{
I SHARMA $^{\mathrm{a}}$, A SAXENA ${ }^{\mathrm{a}}, \mathrm{C} \mathrm{K}$ OJHA $^{\mathrm{a}}$, P PARDASANI ${ }^{\mathrm{a}}$, \\ R T PARDASANI ${ }^{\mathrm{a} *}$ and T MUKHERJEE ${ }^{\mathrm{b}}$ \\ ${ }^{a}$ Department of Chemistry, University of Rajasthan, Jaipur 302 004, India \\ ${ }^{\mathrm{b}}$ Radiation Chemistry and Chemical Dynamics Division, Bhabha Atomic \\ Research Centre, Mumbai 400 085, India \\ e-mail: pardasanirt@ satyam.net.in
}

\begin{abstract}
The reactions of indol-2,3-dione derivatives with 3-phenyl-5-isoxazolone, 2-thiazoline-2-thiol, 1-phenyl-3-methyl-5-pyrazolone under photochemical conditions have been described. The UV light-induced irradiation mainly produced benzazepine and quinoline carboxylic acid derivatives. The products have been characterized on the basis of spectral data and elemental analyses.
\end{abstract}

Keywords. Indol-2,3-dione; 3-phenyl-5-isoxazolone; 2-thiazoline-2-thiol; 1-phenyl3-methyl-5-pyrazolone; photochemical irradiation; spectral characterization.

\section{Introduction}

Indol-2,3-dione (isatin) derivatives 1a-c have been long known for a wide spectrum of biological activities. ${ }^{1-3}$ It is a core constituent of many naturally occurring alkaloids and drugs. We have investigated the photochemical reactions of indol-2,3-dione derivatives with various five-membered heterocycles containing $\mathrm{O}-\mathrm{C}-\mathrm{N}, \quad \mathrm{N}-\mathrm{C}-\mathrm{S}$ and $\mathrm{N}-\mathrm{C}-\mathrm{N}$ linkages. All these nuclei viz. 3-phenyl-5-isoxazolone, ${ }^{4,5}$ 2, 2-thiazoline-2-thiol ${ }^{6,7} \mathbf{3}$ and 1phenyl-3-methyl-5-pyrazolone 4 are well recognized for their pharmacological activities. Thus, emergent molecules from the reaction between $\mathbf{1}$ and 2-4 may be associated with wide spectrum of biological activities. Keeping in view the observation of Haucke et $a l^{8}$ that under photochemical conditions, indol-2,3-dione decomposes to isatic acid $\mathbf{5}$ which may react through various intermediates coupled with the fact that reacting fivemembered heterocycles may exist in different tautomeric forms and can react via multiple pathways, we have investigated the photo-reactions of indol-2,3-diones with oxazolidine, thiazolidine and pyrazolidine derivatives and the results are presented herein.

\section{Experimental}

Melting points were determined in open glass capillaries and were uncorrected. The IR spectra were recorded on Nicolet Magna $\mathrm{IR}^{\mathrm{TM}}$ spectrometer Model 550 in $\mathrm{KBr}$ pellets. The ${ }^{1} \mathrm{H}$ NMR and ${ }^{13} \mathrm{C}$ NMR spectra were obtained in $\mathrm{CDCl}_{3}$ and DMSO- $d_{6}$ on a FX 90Q Jeol spectrometer at $300 \mathrm{MHz}$ and $89.55 \mathrm{MHz}$, respectively, using TMS as an internal

\footnotetext{
*For correspondence
} 
standard. Microanalyses were obtained using a Perkin-Elmer series $11 \mathrm{C}, \mathrm{H}, \mathrm{N}, \mathrm{S}$ and $\mathrm{O}$ Analyser-2400. Photochemical irradiation was conducted under nitrogen atmosphere by a Hanovia $1 \mathrm{~L}$ photochemical reactor equipped with a medium pressure mercury arc lamp $(298-310 \mathrm{~nm})$. The solvents were purified by standard procedures. ${ }^{11,12}$

\subsection{Typical method for photochemical reactions}

A mixture of 5-bromoisatin $1 \mathbf{b}(0.81 \mathrm{~g} ; 3.3 \mathrm{mmol})$ and 3-phenyl-5-isoxazolone 2 (1.07 g; $6.6 \mathrm{mmol})$ in the molar ratio of $1: 2$ in dry THF $(190 \mathrm{ml})$ was subjected to UV irradiation in the photochemical reactor under an inert atmosphere for $48 \mathrm{~h}$. The reaction was monitored till complete consumption of 5-bromoisatin. The mixture was then concentrated under vacuum and subjected to column chromatography over silica gel. Two major fractions were obtained. The first fraction from petroleum ether-chloroform $(1: 3)$ gave compound $\mathbf{6 b}$ as a dark violet solid $\left(0.66 \mathrm{~g}, 35 \%, \mathrm{mp} 180^{\circ} \mathrm{C}\right)$ whereas another fraction from ethylacetate-methanol $(9: 1)$ afforded $7 \mathbf{b}$ as a light brown solid $(0.27 \mathrm{~g}, 15 \%$, $\left.\mathrm{mp} 199^{\circ} \mathrm{C}\right)$.

\section{Results and discussion}

The reaction of isatin 1a-b with 3-phenyl-5-isoxazolone $\mathbf{2}$ is shown in scheme 1. Two products characterized as 4,5-dioxo-3-phenylisoxazolo[5,4-b]benzazepine $\mathbf{6}$ and 3-phenylisoxazolo[5,4-b]quinoline-4-carboxylic acid 7 were obtained in $35 \%$ and $15 \%$ yield (scheme 1).

The formation of compound $\mathbf{6}$ may be explained by the condensation of isatic acid $\mathbf{5}$ with enolic form of isoxazolone, while compound 7 may arise by the condensation of the<smiles>[R]c1ccc2c(c1)C(=O)C(=O)N2</smiles>

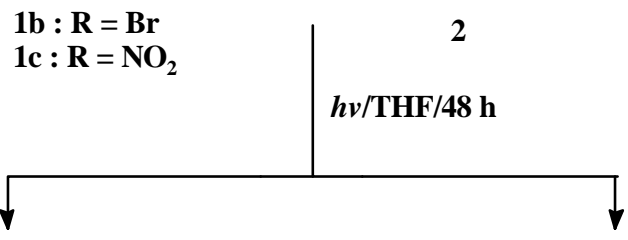<smiles>[R]c1ccc2[nH]c3onc(-c4ccccc4)c3c(=O)c(=O)c2c1</smiles><smiles>[R]c1ccc2nc3onc(-c4ccccc4)c3c(C(=O)O)c2c1</smiles>

6b-c

$7 b-c$

Scheme 1. Photochemical condensation of isatin with 3-phenyl-5-isoxazolone. 
keto group of isatic acid $\mathbf{5}$ with the active methylene group of isoxazolone, followed by cyclization (scheme 2).

The fact that isatin decomposes to isatic acid under photochemical conditions has been supported by molecular modelling using PC PH4 programme. Results of the calculation of the heat of formation have been summarised in table 1 .

The photochemical irradiation of isatin derivatives 1a-c with 2-thiazoline-2-thiol 3 also produced two products which were characterized as 2,4'-dehydro-2-[2'-mercaptothiazolidine]indol-3-one $\mathbf{8}$ and 2-mercaptothiazolo[5,4-b]quinoline-4-carboxylic acid $\mathbf{9}$ in $50 \%$ and $20 \%$ yield respectively (scheme 3 ).<smiles>[R]c1ccc2c(c1)C(=O)C(=O)N2</smiles>

$1 \mathrm{~b}: \mathbf{R}=\mathbf{B r}$ $1 c: \mathbf{R}=\mathrm{NO}_{2}$<smiles>[R]c1ccc(N)c(C(=O)C(=O)O)c1</smiles><smiles>Oc1cc(-c2ccccc2)no1</smiles><smiles>[R]c1ccc2[nH]c3onc(-c4ccccc4)c3c(=O)c2c1</smiles>

6b-c<smiles>[R]c1ccc(N)c(C(=O)C(=O)O)c1</smiles>

5<smiles>O=C1CC(c2ccccc2)=NO1</smiles>

2<smiles>[R]c1ccc2nc3onc(-c4ccccc4)c3c(C(=O)O)c2c1</smiles>

7b-c

Scheme 2. Mechanism for the formation of the photo-products 6 and 7.

Table 1. Heat of formation of indol-2,3-diones and the corresponding isatic acids.

\begin{tabular}{lcccc}
\hline Compound & 5-Bromoisatin & 5-Bromoisatic acid & 5-Nitroisatin & 5-Nitroisatic acid \\
\hline$H_{f}\left(\mathrm{kcal} / \mathrm{mol}^{-1}\right)$ & +9.80 & -35.52 & +31.78 & -10.19 \\
\hline
\end{tabular}


The formation of $\mathbf{8}$ may be explained through the intermediacy of diketone resulting from the condensation of isatic acid 5 with 2-thiazoline-2-thiol. The intramolecular nucleophilic attack of the amino group on the carbonyl carbon and subsequent dehydration results in 8 (scheme 4).

Compound 9 (see scheme 3) may arise by the coupling of keto group of isatic acid $\mathbf{5}$ with methylene of 2-thiazoline-2-thiol followed by cyclisation. Such functionalized thiazolinoquinolines 9 may serve as useful synthons for the total synthesis of naturally occurring quinoline alkaloids. ${ }^{9}$

When the reaction of isatin 1a was carried out with pyrazolone 4 under photochemical irradiation, 3-methyl-4,5-dioxo-1-phenylpyrazolo [3,4-b]benzazepine $\mathbf{1 0}$ was obtained as<smiles>[R]c1ccc2c(c1)C(=O)C(=O)N2</smiles>

$1 \mathbf{a}: \mathbf{R}=\mathbf{H}$

$\mathbf{1 b}: \mathbf{R}=\mathbf{B r}$

1c $: \mathbf{R}=\mathrm{NO}_{2}$<smiles>SC1=NCCS1</smiles>

$h v / \mathrm{THF} / 48 \mathrm{~h}$<smiles>[R]c1ccc2nc3sc(S)nc3c(C(=O)O)c2c1</smiles>

$\mathbf{8 a}-\mathbf{c}$

Scheme 3. Photo-condensation of isatin with 2-thiazoline-2-thiol.<smiles>[R]C1=CC2C(=O)C(=O)NC2C=C1</smiles><smiles>[R]c1ccc(N)c(C(=O)C(=O)O)c1</smiles><smiles>[CH]1CC2CCC1S2</smiles><smiles>[R]c1ccc(N)c(C(=O)C(=O)C2CSC(S)=N2)c1</smiles>

1a: $\mathbf{R}=\mathbf{H}$ $1 \mathrm{~b}: \mathbf{R}=\mathbf{B r}$ $1 c: \mathrm{R}=\mathrm{NO}_{2}$

5<smiles>[R]c1ccc2c(c1)C(O)/C(=C1/CSC(S)=N1)N2</smiles><smiles>[3H]C</smiles><smiles>[R]c1ccc2c(c1)[C@H](O)[C@@](O)([C@H]1CSC(S)=N1)N2</smiles>

Scheme 4. Mechanism for the formation of the product 8 . 
a major product in $32 \%$ yield (scheme 5). Analogous reaction of $\mathbf{1 b}$ with $\mathbf{4}$ furnished 3[2'-(1"-carboxy-2"-oxoethyl]-4'-fluorophenyl]-5-fluoroindol-2-one $\mathbf{1 1}$ in $42 \%$ yield along with minor products 12 and 13 (scheme 6). The compound $\mathbf{1 0}$ is formed in the similar fashion as compound $\mathbf{6}$ whereas $\mathbf{1 1}$ may arise by the condensation of amino group of isatic acid with one of the keto group of isatin.<smiles>O=C1Nc2ccccc2C1=O</smiles>

1a<smiles>CC1=NN(c2ccccc2)C(=O)C1</smiles>

4

Scheme 5. Photo-condensation reaction of isatin with pyrazolone.

1d

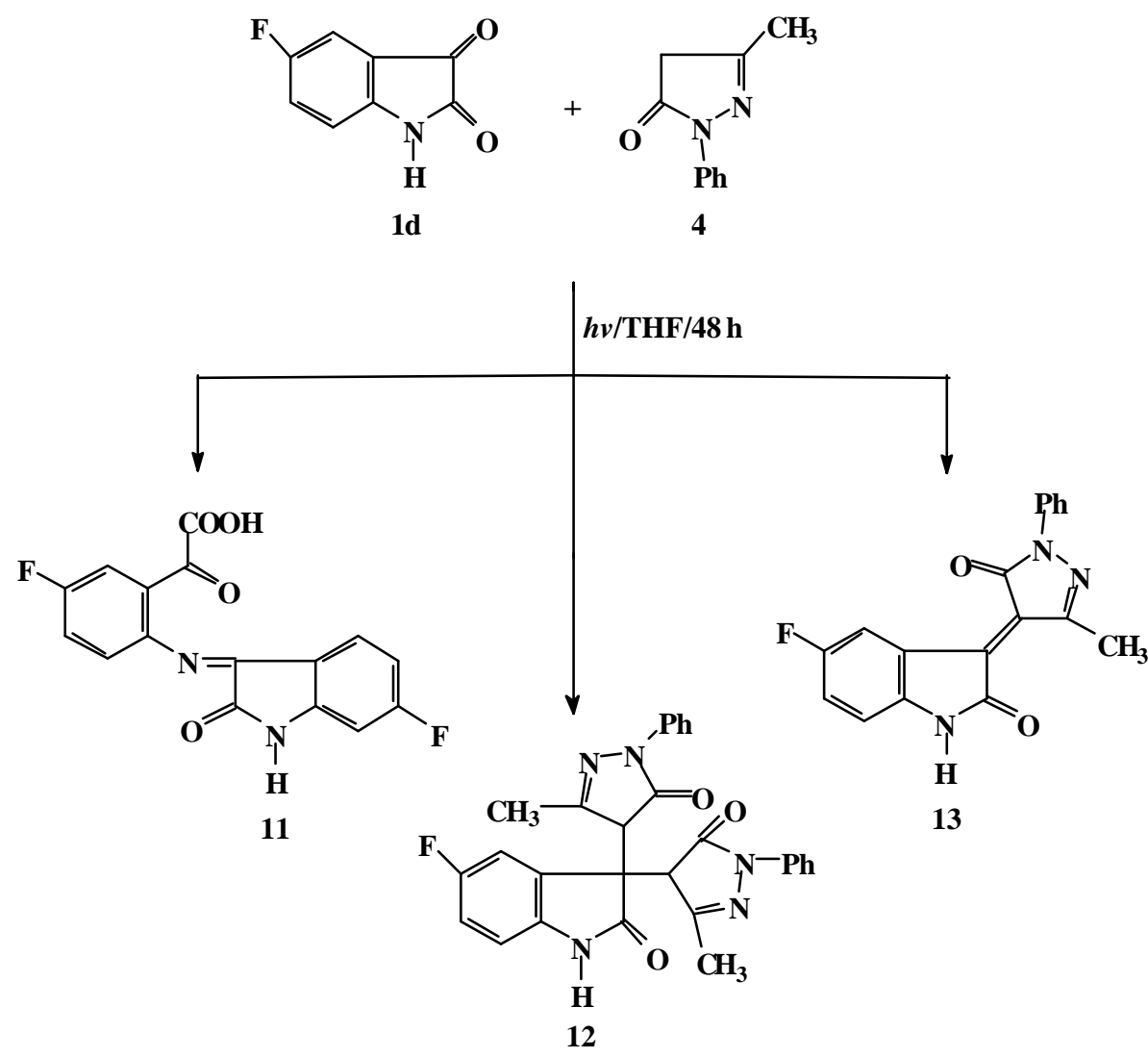

10<smiles>Cc1nn(-c2ccccc2)c2[nH]c3ccccc3c(=O)c12</smiles>

Scheme 6. Photo-condensation reaction of 5-fluoroisatin with pyrazolone. 


\section{Spectral studies}

In IR spectra of products $\mathbf{6 a - b}, \mathbf{8 a - c}, \mathbf{1 0}$ and $\mathbf{1 1}$ the characteristic absorption band for the imino group was present at $3230-3190 \mathrm{~cm}^{-1}$. The absorption peak expected from the carbonyl group was observed in all the photo-products at $1760-1690 \mathrm{~cm}^{-1}$. The absorption peak for $>\mathrm{C}=\mathrm{N}$ in quinoline carboxylic acids $\mathbf{7 a}-\mathbf{b}$ and $\mathbf{9 a - c}$ was seen at $1610 \mathrm{~cm}^{-1}$. The peak at $2715 \mathrm{~cm}^{-1}$ corresponding to $\mathrm{SH}$ group was found in compounds $8 \mathbf{a}-\mathbf{c}$ and 9a-c.

In ${ }^{1} \mathrm{H}$ NMR spectra of all the photo-products aromatic protons were seen in the normal range i.e. $\delta \quad 6 \cdot 0-8.5 \mathrm{ppm}$. A singlet around $\delta \quad 9.2-10.5 \mathrm{ppm}$ corresponded to imino protons in all the products. Carboxylic proton in $\mathbf{7 a - b}$ and $\mathbf{9 a - c}$ appeared as broad singlet at $\delta 11.0$ and $12.2 \mathrm{ppm}$ respectively. The ${ }^{19} \mathrm{~F}$ NMR of compound 11 showed two signals at $\delta-118.9$ and $-119.6 \mathrm{ppm}$ indicating that two $\mathrm{F}$ atoms are present in this compound and

Table 2. Physical and analytical data of photo-products.

\begin{tabular}{|c|c|c|c|c|c|c|c|}
\hline \multirow{2}{*}{$\begin{array}{l}\text { Compd. } \\
\text { no. }\end{array}$} & \multirow{2}{*}{$\begin{array}{l}\text { Physical } \\
\text { state }\end{array}$} & \multirow{2}{*}{$\begin{array}{l}\text { Molecular } \\
\text { formula }\end{array}$} & \multirow{2}{*}{$\begin{array}{l}\mathrm{MP} \\
\left({ }^{\circ} \mathrm{C}\right)\end{array}$} & \multirow{2}{*}{$\begin{array}{l}\text { Yield } \\
(\%)\end{array}$} & \multicolumn{3}{|c|}{$\begin{array}{l}\text { Elemental analyses } \\
\text { calculated (found) }\end{array}$} \\
\hline & & & & & $\mathrm{C}$ & $\mathrm{H}$ & $\mathrm{N}$ \\
\hline $6 b$ & Violet solid & $\mathrm{C}_{17} \mathrm{H}_{9} \mathrm{~N}_{2} \mathrm{O}_{3} \mathrm{Br}$ & 180 & 35 & $\begin{array}{l}55 \cdot 28 \\
(55 \cdot 26)\end{array}$ & $\begin{array}{l}2 \cdot 43 \\
(2 \cdot 40)\end{array}$ & $\begin{array}{l}7.58 \\
(7.57)\end{array}$ \\
\hline $6 c$ & Brick red solid & $\mathrm{C}_{17} \mathrm{H}_{9} \mathrm{~N}_{3} \mathrm{O}_{5}$ & 190 & 28 & $\begin{array}{c}60 \cdot 89 \\
(60 \cdot 85)\end{array}$ & $\begin{array}{l}2 \cdot 68 \\
(2 \cdot 64)\end{array}$ & $\begin{array}{l}12.53 \\
(12 \cdot 49)\end{array}$ \\
\hline $7 b$ & Brown solid & $\mathrm{C}_{17} \mathrm{H}_{9} \mathrm{~N}_{2} \mathrm{O}_{3} \mathrm{Br}$ & 199 & 15 & $\begin{array}{l}55 \cdot 28 \\
(55 \cdot 25)\end{array}$ & $\begin{array}{l}2 \cdot 43 \\
(2 \cdot 40)\end{array}$ & $\begin{array}{c}7 \cdot 58 \\
(7 \cdot 56)\end{array}$ \\
\hline $7 c$ & Yellowish solid & $\mathrm{C}_{17} \mathrm{H}_{9} \mathrm{~N}_{3} \mathrm{O}_{5}$ & 110 & 18 & $\begin{array}{c}60 \cdot 89 \\
(60 \cdot 87)\end{array}$ & $\begin{array}{l}2 \cdot 68 \\
(2 \cdot 64)\end{array}$ & $\begin{array}{c}12.53 \\
(12.53)\end{array}$ \\
\hline $8 \mathbf{a}$ & Orange solid & $\mathrm{C}_{11} \mathrm{H}_{8} \mathrm{~N}_{2} \mathrm{OS}_{2}$ & 190 & 50 & $\begin{array}{c}53 \cdot 22 \\
(53 \cdot 18)\end{array}$ & $\begin{array}{c}3 \cdot 22 \\
(3 \cdot 19)\end{array}$ & $\begin{array}{l}11.29 \\
(11.22)\end{array}$ \\
\hline $8 b$ & Red solid & $\mathrm{C}_{11} \mathrm{H}_{7} \mathrm{~N}_{2} \mathrm{OS}_{2} \mathrm{Br}$ & 230 & 52 & $\begin{array}{c}40 \cdot 36 \\
(40 \cdot 32)\end{array}$ & $\begin{array}{l}2 \cdot 14 \\
(2 \cdot 11)\end{array}$ & $\begin{array}{c}8 \cdot 56 \\
(8.48)\end{array}$ \\
\hline $8 c$ & Yellow solid & $\mathrm{C}_{11} \mathrm{H}_{7} \mathrm{~N}_{3} \mathrm{O}_{3} \mathrm{~S}_{2}$ & 240 & 45 & $\begin{array}{c}45 \cdot 05 \\
(45 \cdot 00)\end{array}$ & $\begin{array}{c}2 \cdot 38 \\
(2 \cdot 32)\end{array}$ & $\begin{array}{c}14 \cdot 32 \\
(14 \cdot 26)\end{array}$ \\
\hline $9 a$ & Yellow solid & $\mathrm{C}_{11} \mathrm{H}_{6} \mathrm{~N}_{2} \mathrm{O}_{2} \mathrm{~S}_{2}$ & 180 & 18 & $\begin{array}{c}50 \cdot 38 \\
(50 \cdot 32)\end{array}$ & $\begin{array}{l}2 \cdot 29 \\
(2 \cdot 23)\end{array}$ & $\begin{array}{c}10.68 \\
(10.68)\end{array}$ \\
\hline $9 b$ & Red solid & $\mathrm{C}_{11} \mathrm{H}_{5} \mathrm{~N}_{2} \mathrm{O}_{2} \mathrm{~S}_{2} \mathrm{Br}$ & 210 & 15 & $\begin{array}{c}38 \cdot 72 \\
(38 \cdot 70)\end{array}$ & $\begin{array}{c}1.46 \\
(1.41)\end{array}$ & $\begin{array}{c}8 \cdot 21 \\
(8 \cdot 19)\end{array}$ \\
\hline $9 \mathrm{c}$ & Yellow solid & $\mathrm{C}_{11} \mathrm{H}_{5} \mathrm{~N}_{3} \mathrm{O}_{4} \mathrm{~S}_{2}$ & 235 & 20 & $\begin{array}{c}42 \cdot 99 \\
(42.94)\end{array}$ & $\begin{array}{c}1 \cdot 62 \\
(1.57)\end{array}$ & $\begin{array}{c}13 \cdot 6 \\
(13 \cdot 4)\end{array}$ \\
\hline 10 & Orange crystals & $\mathrm{C}_{18} \mathrm{H}_{13} \mathrm{~N}_{3} \mathrm{O}_{2}$ & 186 & 32 & $\begin{array}{l}71 \cdot 28 \\
(71 \cdot 35)\end{array}$ & $\begin{array}{c}4 \cdot 29 \\
(4 \cdot 20)\end{array}$ & $\begin{array}{c}13.86 \\
(13.79)\end{array}$ \\
\hline 11 & Yellow & $\mathrm{C}_{18} \mathrm{H}_{8} \mathrm{~N}_{2} \mathrm{O}_{4} \mathrm{~F}_{2}$ & 206 & 42 & $\begin{array}{l}58 \cdot 18 \\
(58 \cdot 07)\end{array}$ & $\begin{array}{c}2 \cdot 42 \\
(2 \cdot 33)\end{array}$ & $\begin{array}{c}8.48 \\
(8.41)\end{array}$ \\
\hline 12 & White crystals & $\mathrm{C}_{28} \mathrm{H}_{22} \mathrm{~N}_{5} \mathrm{O}_{3} \mathrm{~F}$ & 168 & 13 & $\begin{array}{c}67 \cdot 87 \\
(67 \cdot 78)\end{array}$ & $\begin{array}{c}4 \cdot 44 \\
(4 \cdot 52)\end{array}$ & $\begin{array}{c}14 \cdot 14 \\
(14 \cdot 21)\end{array}$ \\
\hline 13 & Violet solid & $\mathrm{C}_{18} \mathrm{H}_{12} \mathrm{~N}_{3} \mathrm{O}_{2} \mathrm{~F}$ & 234 & 7 & $\begin{array}{c}67 \cdot 29 \\
(67 \cdot 21)\end{array}$ & $\begin{array}{c}3 \cdot 72 \\
(3 \cdot 67)\end{array}$ & $\begin{array}{c}13 \cdot 08 \\
(13 \cdot 01)\end{array}$ \\
\hline
\end{tabular}


Table 3. Spectral data of photo-condensation products.

\begin{tabular}{|c|c|c|}
\hline $\begin{array}{l}\text { Compd. } \\
\text { no. }\end{array}$ & $\operatorname{IR}\left(\mathrm{cm}^{-1}\right)$ & ${ }^{1} \mathrm{H}$ NMR $(\delta \mathrm{ppm})$ \\
\hline 6b & $\begin{array}{l}3230(s, \mathrm{NH}), 1760,1740 \\
\quad(\alpha \text {-diketone })\end{array}$ & $\begin{array}{l}7 \cdot 0(d, \mathrm{~J}=6 \cdot 0 \mathrm{~Hz}, \mathrm{H}-9), 7 \cdot 3-7 \cdot 4\left(m, 3-\mathrm{C}_{6} \mathrm{H}_{5}\right) \\
8 \cdot 1\left(d d, J_{1}=7 \cdot 0 \mathrm{~Hz}, J_{2}=2 \cdot 5 \mathrm{~Hz}, \mathrm{H}-8\right), 8 \cdot 23 \\
(d, J=2 \cdot 5 \mathrm{~Hz}, \mathrm{H}-6), 9 \cdot 8(s, \mathrm{NH})\end{array}$ \\
\hline $6 c$ & $\begin{array}{l}3228(s, \mathrm{NH}), 1758,1740 \\
\quad(\alpha \text {-diketone })\end{array}$ & $\begin{array}{c}6.8(d, J=6.0 \mathrm{~Hz}, \mathrm{H}-9), 7.4\left(m, 3-\mathrm{C}_{6} \mathrm{H}_{5}\right), 8 \cdot 15 \\
\left(d d, J_{1}=7 \cdot 0 \mathrm{~Hz}, J_{2}=2.5 \mathrm{~Hz}, \mathrm{H}-8\right), 8 \cdot 20 \\
(d, J=2.5 \mathrm{~Hz}, \mathrm{H}-6), 9.9(s, \mathrm{NH})\end{array}$ \\
\hline $7 \mathbf{b}$ & $\begin{array}{l}3590(s, b r, \mathrm{OH}), 1690(s,>\mathrm{C}=\mathrm{O}) \\
\quad 1610(s, \mathrm{C}=\mathrm{N})\end{array}$ & $\begin{array}{l}6.97(d, J=7 \cdot 0 \mathrm{~Hz}, \mathrm{H}-8), 7 \cdot 44\left(m, 3-\mathrm{C}_{6} \mathrm{H}_{5}\right) \\
\quad 8 \cdot 11\left(d d, J_{1}=7 \cdot 0 \mathrm{~Hz}, J_{2}=2 \cdot 0 \mathrm{~Hz}, \mathrm{H}-7\right), 8 \cdot 2 \\
\quad(d, J=2 \cdot 0 \mathrm{~Hz}, \mathrm{H}-5)), 9 \cdot 9(s, \mathrm{NH})\end{array}$ \\
\hline 7c & $\begin{array}{l}3600(s, b r, \mathrm{OH}), 1710(s,>\mathrm{C}=\mathrm{O}) \\
\quad 1610(s, \mathrm{C}=\mathrm{N})\end{array}$ & $\begin{array}{l}6.99(d, J=6 \cdot 5 \mathrm{~Hz}, \mathrm{H}-8), 7 \cdot 34-7 \cdot 45(m, \\
\left.3-\mathrm{C}_{6} \mathrm{H}_{5}\right), 8 \cdot 20\left(d d, J_{1}=6 \cdot 5 \mathrm{~Hz}, J_{2}=2 \cdot 0 \mathrm{~Hz},\right. \\
\mathrm{H}-7), 8 \cdot 25(d, J=2 \cdot 0 \mathrm{~Hz}, \mathrm{H}-5)), 11 \cdot 35 \\
(s, \mathrm{COOH})\end{array}$ \\
\hline $8 \mathbf{a}$ & $\begin{array}{l}3320(s, \mathrm{NH}), 2910\left(s h, \mathrm{CH}_{2}\right), 2715 \\
\quad(s, \mathrm{SH}), 1690(s,>\mathrm{C}=\mathrm{O})\end{array}$ & $\begin{array}{l}3 \cdot 70\left(s, \mathrm{CH}_{2}\right), 6 \cdot 97-8 \cdot 2(m, \mathrm{Ar}-\mathrm{H}), 8 \cdot 50 \\
\quad(s, \mathrm{NH}), 11 \cdot 1(s, \mathrm{SH})\end{array}$ \\
\hline $\mathbf{8 b}$ & $\begin{array}{l}3290(s, \mathrm{NH}), 2970\left(s h, \mathrm{CH}_{2}\right), 2735 \\
\quad(s, \mathrm{SH}), 1710(s,>\mathrm{C}=\mathrm{O})\end{array}$ & $\begin{array}{l}3.65\left(s, \mathrm{CH}_{2}\right), 6 \cdot 90-8 \cdot 4(m, \mathrm{Ar}-\mathrm{H}), 9 \cdot 3 \\
\quad(s, \mathrm{NH}), 11 \cdot 0(\mathrm{~s}, \mathrm{SH})\end{array}$ \\
\hline $8 c$ & $\begin{array}{l}3350(s, \mathrm{NH}), 2980\left(s h, \mathrm{CH}_{2}\right), 2785 \\
\quad(s, \mathrm{SH}), 1730(s,>\mathrm{C}=\mathrm{O}), 1610(m, \\
\quad \mathrm{C}=\mathrm{N}), 1575,1360\left(m, \mathrm{NO}_{2}\right)\end{array}$ & $\begin{array}{l}3 \cdot 72\left(s, \mathrm{CH}_{2}\right), 6 \cdot 83-8 \cdot 12(m, \mathrm{Ar}-\mathrm{H}), 9 \cdot 25 \\
\quad(s, \mathrm{NH}), 11 \cdot 4(s, \mathrm{SH})\end{array}$ \\
\hline $9 \mathbf{a}$ & $\begin{array}{l}3560(s, \mathrm{OH}), 2810(s, \mathrm{SH}), 1760 \\
\quad(s,>\mathrm{C}=\mathrm{O}), 1630(\mathrm{C}=\mathrm{N})\end{array}$ & $\begin{array}{l}7 \cdot 3-8 \cdot 81(m, \mathrm{Ar}-\mathrm{H}), 11 \cdot 2(s, \mathrm{SH}), 12 \cdot 2 \\
\quad(s, \mathrm{COOH})\end{array}$ \\
\hline $9 b$ & $\begin{array}{l}3520(s, \mathrm{OH}), 2800(s, \mathrm{SH}), 1740 \\
\quad(s,>\mathrm{C}=\mathrm{O}), 1610(\mathrm{C}=\mathrm{N})\end{array}$ & $\begin{array}{l}\text { 7.4-8.5 }(m, \mathrm{Ar}-\mathrm{H}), 11 \cdot 0(\mathrm{~s}, \mathrm{SH}), 12 \cdot 5 \\
\quad(s, \mathrm{COOH})\end{array}$ \\
\hline $9 \mathrm{c}$ & $\begin{array}{l}3550(s, \mathrm{OH}), 2790(s, \mathrm{SH}), 1730 \\
\quad(s,>\mathrm{C}=\mathrm{O}), 1620(\mathrm{C}=\mathrm{N}), 1570 \\
1400\left(m, \mathrm{NO}_{2}\right)\end{array}$ & $\begin{array}{l}7 \cdot 35-8 \cdot 21(m, \mathrm{Ar}-\mathrm{H}), 11 \cdot 4(s, \mathrm{SH}), 12 \cdot 25 \\
\quad(s, \mathrm{COOH})\end{array}$ \\
\hline 10 & $\begin{array}{l}3230(s, \mathrm{NH}), 1760,1740 \\
\quad(s,>\mathrm{C}=\mathrm{O}), 1630(m, \mathrm{C}=\mathrm{N})\end{array}$ & $2 \cdot 1\left(s, \mathrm{CH}_{3}\right), 6 \cdot 5-7 \cdot 2(m, \mathrm{Ar}-\mathrm{H}), 9 \cdot 35(s, \mathrm{NH})$ \\
\hline 11 & $\begin{array}{l}3480(s, \mathrm{OH}), 3180(s, \mathrm{NH}), 1770, \\
\quad 1750,1720(s, \mathrm{C}=\mathrm{O})\end{array}$ & $\begin{array}{l}\text { 7.15-8.32 }(m, \mathrm{Ar}-\mathrm{H}), 9 \cdot 4(s, \mathrm{NH}), 11 \cdot 0 \\
\quad(s, \mathrm{COOH})\end{array}$ \\
\hline 12 & $\begin{array}{c}3350(s, \mathrm{NH}), 1730,1700,1690 \\
(s,>\mathrm{C}=\mathrm{O}), 1630(m, \mathrm{C}=\mathrm{N})\end{array}$ & $\begin{array}{l}2 \cdot 1\left(s, 2 \times \mathrm{CH}_{3}\right), 3 \cdot 8(m, 2 \times \mathrm{CH}), 6 \cdot 8-7 \cdot 4 \\
\quad(m, \mathrm{Ar}-\mathrm{H}), 9 \cdot 3(\mathrm{~s}, \mathrm{NH})\end{array}$ \\
\hline 13 & $\begin{array}{l}3260-3220(s, \mathrm{NH}), 1700,1680 \\
\quad(>\mathrm{C}=\mathrm{O}), 1615(\mathrm{C}=\mathrm{C})\end{array}$ & $1.9\left(s, \mathrm{CH}_{3}\right), 6 \cdot 8-7 \cdot 4(m, \mathrm{Ar}-\mathrm{H}), 9 \cdot 3(s, \mathrm{NH})$ \\
\hline
\end{tabular}

are in different environments. A broad hump at $\delta 11.1$ for SH proton was present in $\mathbf{8 a - c}$ and 9a-c. In ${ }^{13} \mathrm{C}$ NMR of the product $6 \mathbf{a}$, the carbonyl carbon appeared at $\delta 181.9, \mathrm{C}-2^{\prime}$ appeared at $\delta 158 \cdot 3$, benzenoid carbons appeared in the range of $\delta$ 147.85-126.3, olefinic carbons (C-2 and $\left.\mathrm{C}-5^{\prime}\right)$ appeared at $\delta 126.3$ and methylene carbon appeared at $\delta 112.8$ ppm. In ${ }^{1} \mathrm{H}$ NMR of compounds 12 and 13, signals corresponding to the active methylene protons of pyrazolone 4 were absent in the region of $\delta 4.14$ whereas methyl, aromatic and imino protons were present in their required region. Additional evidence was obtained from the mass spectra of 10, 11, 12 and 13 in which $M^{+}$peaks were seen at $303(8 \%), 303$ 
Table 4. Antimicrobial activity of the photo-products $8 \mathbf{8}-\mathbf{c}$ and $9 \mathbf{9 a - c}$.

\begin{tabular}{|c|c|c|c|c|c|}
\hline \multirow{2}{*}{$\begin{array}{l}\text { Compd. } \\
\text { no. }\end{array}$} & \multicolumn{5}{|c|}{ Zone of inhibition in mm (activity index) } \\
\hline & E. coli & S. facaelus & R. solani & F. oxysporium & F. solani \\
\hline $8 \mathbf{a}$ & $\begin{array}{l}8 \cdot 9 \\
(0 \cdot 88)\end{array}$ & $\begin{array}{l}9 \cdot 4 \\
(0 \cdot 95)\end{array}$ & $\begin{array}{l}10 \cdot 2 \\
(1 \cdot 02)\end{array}$ & $\begin{array}{c}8 \cdot 4 \\
(0 \cdot 84)\end{array}$ & $\begin{array}{l}7 \cdot 9 \\
(0 \cdot 78)\end{array}$ \\
\hline $8 \mathbf{b}$ & $\begin{array}{l}11 \cdot 2 \\
(1 \cdot 12)\end{array}$ & $\begin{array}{c}9 \cdot 8 \\
(1 \cdot 02)\end{array}$ & $\begin{array}{l}12 \cdot 6 \\
(1 \cdot 04)\end{array}$ & $\begin{array}{l}9 \cdot 8 \\
(0 \cdot 92)\end{array}$ & $\begin{array}{l}10 \cdot 6 \\
(1 \cdot 06)\end{array}$ \\
\hline $8 \mathbf{c}$ & $\begin{array}{l}13 \cdot 4 \\
(1 \cdot 10)\end{array}$ & $\begin{array}{l}11 \cdot 6 \\
(1 \cdot 12)\end{array}$ & $\begin{array}{l}12 \cdot 6 \\
(1 \cdot 02)\end{array}$ & $\begin{array}{l}13 \cdot 0 \\
(1 \cdot 13)\end{array}$ & $\begin{array}{l}12 \cdot 2 \\
(1 \cdot 12)\end{array}$ \\
\hline $9 \mathbf{a}$ & $\begin{array}{l}10 \cdot 0 \\
(1 \cdot 00)\end{array}$ & $\begin{array}{l}9 \cdot 1 \\
(0 \cdot 98)\end{array}$ & $\begin{array}{l}9.8 \\
(1.07)\end{array}$ & $\begin{array}{l}12 \cdot 2 \\
(1 \cdot 05)\end{array}$ & $\begin{array}{l}11 \cdot 0 \\
(1 \cdot 10)\end{array}$ \\
\hline $9 b$ & $\begin{array}{l}10 \cdot 5 \\
(1 \cdot 05)\end{array}$ & $\begin{array}{l}9 \cdot 8 \\
(0 \cdot 98)\end{array}$ & $\begin{array}{l}10 \cdot 0 \\
(1 \cdot 02)\end{array}$ & $\begin{array}{l}12 \cdot 0 \\
(1 \cdot 06)\end{array}$ & $\begin{array}{l}12 \cdot 6 \\
(1 \cdot 08)\end{array}$ \\
\hline $9 \mathrm{c}$ & $\begin{array}{l}11 \cdot 5 \\
(1 \cdot 15)\end{array}$ & $\begin{array}{l}10 \cdot 5 \\
(1 \cdot 05)\end{array}$ & $\begin{array}{l}11 \cdot 4 \\
(1 \cdot 12)\end{array}$ & $\begin{array}{l}12 \cdot 5 \\
(1 \cdot 20)\end{array}$ & $\begin{array}{l}13 \cdot 5 \\
(1 \cdot 20)\end{array}$ \\
\hline
\end{tabular}

Activity index = inhibition zone of the sample/inhibition zone of the standard

(20\%), 495 (12\%) and 321 (52\%), respectively. All these assignments are in harmony with the proposed structures (table 3), which structures were further established by elemental analyses (table 2).

\section{Antimicrobial activity}

Some of the synthesized compounds 8a-c and 9a-c were evaluated for their antimicrobial activities at a concentration of $100 \mu \mathrm{g} / \mathrm{disc}$ in agar media. The method adopted for the estimation of the antimicrobial activity is the paper disc diffusion method of AW Bauer et $a l^{10}$. In this method the compound is allowed to diffuse through a solid medium so that a gradient is established. The test bacterium or fungus is seeded on the medium and its sensitivity to the compound is determined from the inhibition of the growth. Streptomycin and Mycostanin were used as the reference compounds for antibacterial and antifungal activities. The test organisms were E. coli, S. facaelus (bacteria), R. solani, $F$. oxysporium and $F$. solani (fungi) and the results are summarized in table 4.

\section{Conclusions}

(i) On the basis of the calculation of the heat of formation (table 1) it may be concluded that isatic acid is thermodynamically more stable than the corresponding isatin.

(ii) Under photochemical conditions, isatin decomposes to isatic acid (2-amino-phenylglyoxalic acid) whereas there is no such decomposition under thermal conditions.

(iii) There is significant enolization of isoxazolone and pyrazolone under photochemical conditions and this fact is supported by the predominant formation of 6a-b and $\mathbf{1 0}$.

(iv) From table 3, it may be concluded that some of the screened compounds were highly active against the test microorganisms. 


\section{References}

1. Joshi K C and Chand P 1982 Pharmazie 371

2. Benington F, Morin D R and Bradley R J 1976 J. Heterocycl. Chem. 13749

3. Whitehead C W and Whitesitt C A 1974 J. Med. Chem. 171298

4. Tantawy A, Goda F and Abdelal A M 1995 Clin. Pharmcol. J. 47 37; Tantawy A, Goda F and Abdelal A M 1995 Chem. Abstr. 123228016

5. Griesbeck A G, Hill J, Peters K, Peters E M and Schnering H G V 1995 Ann. Chem. 619

6. Moulard J, Lagorce J F, Thomes J C and Raby C 1993 J. Pharm. Pharmacol. 45731

7. Faermark I F, Shvarts G, Grizik S I and Gravik V G 1990 Khim.-Farm. Zh. 2435

8. Hauke G, Seidel B and Grauss A 1987 J. Photochem. 37139

9. Grundon M F 1988 The alkaloids (ed.) A Brossi (San Diego: Academic Press) p. 32

10. Bauwer A W, Kibby W M M, Sherris J C and Turk M 1966 Am. J. Clin. Pathol. 45493

11. Vogel A I 1984 Vogel's textbook of practical organic chemistry 4th edn (London: ELBS Longman)

12. Perrin D D, Armarego W L F and Perrin D R 1980 Purification of laboratory chemicals 2nd edn (Oxford: Pergamon) 